\title{
Singer's high formant associated with different larynx position in styles of singing*
}

\author{
Shiqian Wang**† \\ **Physics Department of Faculty of Science and Music Department of \\ Faculty of Fine Arts, York University, \\ 4700 Keele Street, Downsview, Ontario M3J 1P3, Canada
}

(Received 27 June 1985)

\begin{abstract}
This paper is a further presentation and discussion of the author's voice research and previous paper and film on singer's (high) formant associated with different larynx position in styles of singing. The main results and points are: 1 . The singer's high formant pattern varies with similar characteristic (two to four high and often compact energy peaks, including a possible extra peak $f_{\mathrm{e}}$, in the bright frequency range); 2 . The $f_{\mathrm{e}}$ is also found in high laryngeal singing and does not necessarily appear as a typical acoustic feature in all professional singing voice; 3 . Sundberg's lowered larynx interpretation does not explain the extra formant, singer's formant, and voice quality; 4 . Sundberg's different definitions of singer's formant are not inclusive of the various formant patterns of singing associated with different laryngeal position and different types; 5 . The author also suggests a definition for the singer's formant; 6 . The low laryngeal position could be yet regarded as a distinctive feature for the covered singing voice though not for the trained singing voice; 7 . The source characteristics and the interaction between the source and the supra/sub glottal systems are possibly different in styles of singing associated with various laryngeal positions.
\end{abstract}

PACS number: 43. 70. $-\mathrm{h}, 43.75 .-\mathrm{z}$

\section{INTRODUCTION}

The singer's (high) formant have been studied by Bartholomew, ${ }^{1}$ Rzhevkin, ${ }^{2)}$ Vennard, ${ }^{3)}$ Sundberg, ${ }^{4)}$ and dozens of other researchers.

Bartholomew ${ }^{1)}$ found and defined the singer's (high) formant (hereinafter singer's formant) as a prominent formant which averages around $2.8 \sim 2.9$ $\mathrm{kHz}$ for male voice, and $3.2 \mathrm{kHz}$ for female voice. $\mathrm{He}$ also claimed that the coloraturae sometimes have

* This paper was invited to be presented at the Conference, and originally submitted to the Transactions, of the Committee on Speech Research, Acoustical Society of Japan 1984 (Tokyo).

$\dagger$ Present address: Speech Communication Laboratory, the Institute of Acoustics, Chinese Academy of Science, 5 Zhongguancun Road, Beijing, The People's Republic of China no singer's formant. He explained that the lowered larynx during singing is the source of this singer's formant.

Sundberg ${ }^{4,5)}$ further defined the singing formant as an extra formant in the formants of the singing voice. That is, the singing formant is an extra high spectrum envelope peak near $2.8 \mathrm{kHz}$ located between the third $\left(F_{3}\right)$ and fourth $\left(F_{4}\right)$ formants of vowel sounds produced in male Western opera and concert singing. Sundberg has different meanings for the concept of the singing formant, and we will refer to and discuss those meanings later in this paper. To distinguish this from his other meanings and for the convenience of this discussion the term "extra formant/peak" and its symbol $f_{\mathrm{e}}$ will primarily be used hereafter instead of Sundberg's term "singing formant."

Sundberg ${ }^{4)}$ assumed that lowering the larynx 
"explains* the singing formant and other formant frequency differences between normal speech and male professional singing." That is ${ }^{5)}$ : "The acoustics of the vocal tract when the larynx is lowered are compatible with the generation of just such an extra formant ... if the area of the outlet of the larynx into the pharynx is less than a sixth of the area of the cross section of the pharynx, then the larynx is acoustically mismatched with the rest of the vocal tract," and it ${ }^{5}$ " "acts as a separate resonator capable of adding an extra formant to the transform function of the vocal tract." ${ }_{5}$ ) "... it has a resonance frequency of its own, largely independent of the remainder of the tract. The one-six condition is likely to be met when the larynx is lowered.... The lowering of the larynx, in other words, seems to explain the singing formant peak. ... The result is to shift downward all formant frequencies other than the larynx-dependent extra formant. ... The lowering of the larynx, then, explains not only the singing-formant peak but also major differences in the quality of vowels in speech and singing." Sundberg ${ }^{5}$ claimed that this extra formant "is typical of all voiced sounds sung by professional male singers. Indeed, its presence, regardless of the pitch, the particular vowel and the dynamic level has come to be considered a criterion of quality; the extra peak has been designated the singing formant." Sundberg ${ }^{4)}$ also extended Bartholomew's ${ }^{1,6)}$ conclusion that in the soprano voice "No 'singing formant' is likely to be produced."

Larynx location during singing phonation has been regarded by previous researchers not only as being particularly important in producing the singing formant and to affect the singer's other formants, but also to affect the voice quality and vocal health of the performer. Shipp and Izdebski ${ }^{\text {(7) }}$ found that nonsingers positioned the larynx upward in increased voice frequency and tended to maintain a level at or above the physiological resting position, whereas trained singers maintained laryngeal position below the physiological resting position throughout their vocal range.

In accordance with these findings and explanations another traditional concept has apparently been that singing with a high larynx is generally seen as "singing in the pain" both for the singer (because of its frequent association with vocal deterioration or

* In this paper all italicizations in quoted passages have been added by the present author. damage) and for the listener (because of poor quality of sound produced by some singers) (cf. Refs. $7 \sim 13$ ), etc.).

This author ${ }^{14,15)}$ investigated tenors singing in different styles [some Chinese, early music and Western opera (here, i.e., covered voice)] and his results indicate that revisions to the above concepts, which support some of his previous viewpoints, ${ }^{16)}$ should be made as follows:

1. Laryngeal height is not a reliable way to distinguish a trained singer from an untrained one. Not all trained singers maintain the low laryngeal position.

2. In both high and low laryngeal styles of singing, some trained singers are capable of producing bright healthy voice and its associated acoustic features. Lowering of the larynx therefore does not explain the production of singer's formant and brightness. Singing with a high larynx in and of itself does not necessarily produce a voice of poor quality or cause poor vocal health.

This author and others ${ }^{14,15,17,18)}$ also found that the amplitude and frequency of singer's formant are varied with pitch, vowel and intensity. Recently Sundberg (with Johnson and Wilbrand) ${ }^{19)}$ reported in publication that some sopranos raised their larynges during singing. However it was noticed that Sundberg's concept and his explanation of lowering the larynx for singer's formant were limited to male Western opera and concert singing voice.

\section{QUESTIONS}

In this author's research $(1980 \sim 1983)$ acoustic questions arose concerning the differences between the singer's larynx positions in different styles of male singing.

1. What are the different and common features of the radiated spectra between high and low laryngeal singing voices.

2. What are the different and common features of the radiated spectra between speaking and (high and low laryngeal) singing voice.

3. How do we comment on Sundberg's concept of singer's formant which was interpreted as due to lowering of the larynx and how do we explain the acoustic features associated with high/low laryngeal singing voices.

Those questions should be answered in this research. 


\section{METHOD}

Ten trained tenor voices representive of the Chinese, early music and the Western opera singing style were chosen for this study; each with at least fifteen years of training and experience and in apparent good vocal health. Most of the subjects are soloists and some are highly acclaimed nationwide or regionally (Although these singers may sing in more than one style, and each style, such as the Chinese singing, has many kinds of singing styles, their voices are here only labeled as exhibiting, but not generalizing, either of the three styles). The study consisted of:

(1) Perceptual judgement of timbre.

(2) Measurement, during phonation, of the vertical distance of the larynx from its physiological resting position.

(3) Measurement of acoustic features, including the first five formants, $F_{1}, F_{2}, F_{3}, F_{4}, F_{5}$, the extra formant $f_{\mathrm{e}}$, and the fundamental frequency $F_{0}$.

Perceptual judgements were made by asking each subject to sing (in full voice) three vowels: $/ \mathrm{A} /, / \mathrm{i} /, / \mathrm{u} /$. The sounds produced were rated as to professional brightness on a scale of 1 to 7 by 19 voice teachers and singers at a meeting of the Boston Chapter of the (US) National Association of Teachers of Singing.

Vertical movement of the thyroid cartilage away from its resting position was recorded and measured by the use of photographic slides and videotapes, while the voice was simultaneously recorded on audio tape during phonation. The measurement was facilitated by placing a narrow strip of colored adhesive tape on the skin of the neck, at a right angle to the airway, overlying the tip of the thyroid prominence in the physiological resting position. The extent of the vertical movement as measured on the slides and videotapes was then multiplied by a coefficient obtained by measuring a suitable facial landmark and comparing it to the measurement on the slide or videotape.

Acoustic features were determined by the spectral analysis program on a PDP-11 computer with a UNIX operating system at the Speech Communication Laboratory of MIT, USA. This program was designed to digitize a series of waveform files and perform several kinds of spectral analysis. The formants were determined by using both the Discrete Fourier Transform (DFT) and Linear Predictive Coding (LPC), and the fundamental frequency was obtained from the corresponding cepstrum. The sampling rate was $10 \mathrm{k} / \mathrm{s}$, and the cutoff frequency is $5 \mathrm{kHz}$. The predictor order was 14 . A $25.6 \mathrm{~ms}$ segment of voice preemphasized at $6 \mathrm{~dB} /$ oct was selected visually, multiplied by a Hamming window, and converted into both a magnitude spectrum using the DFT and an idealized spectrum using the all-pole model LPC.

\section{RESULTS}

This paper will deal only with the results of the perceptual judgement of the timbre and the measurement of the larynx position which are related to the following acoustic results and discussion.

1) The voice of all subjects was rated as being in the bright range although, as might be expected, there were different degrees of brightness (see Table 1 rating the brightness of sung vowels).

2) The vowel /i/ and /A/ were judged as being significantly brighter than $/ \mathrm{u} /$ (significance level $p<$ 0.001).

3) In the Chinese and early music style singers, the larynges generally remained above the physiological resting position, especially in the high range of the voice. The larynx of each subject singing in the Western operatic (covered) style was usually below the resting position. This finding was very clearly demonstrated by two subjects who could sing in the Western operatic, and either the Chinese or the early music style (see Fig. 1). While the height of the larynx varied from singer to singer, the position of the larynx was highest for the vowel /i/, nearly as high for $/ \mathrm{A} /$, and the lowest for $/ \mathrm{u} /$ in all styles of singing.

4) In the Chinese and early music singers the height of the larynx increased as the pitch went up. By way of contrast, the Western opera singers' larynges moved progressively lower as pitch went up. These findings held roughly true for pitch up to about $G_{4}$, or $392 \mathrm{~Hz}$ (see Fig. 1).

5) Two to four high energy peaks in some cases including the extra peak $f_{8}$ between or near $F_{8}$, or $F_{4}$,

Table 1 Rating the brightness of sung vowels.

\begin{tabular}{lcccc}
\hline \multicolumn{1}{c}{ Sung vowel } & $\mid \mathrm{A} /$ & $\mid \mathrm{i} /$ & $\mid \mathrm{u} /$ & Average \\
\hline Chinese voice & 5.57 & 5.67 & 5.20 & 5.48 \\
Early music & 4.58 & 4.32 & 4.10 & 4.33 \\
Western opera & 4.60 & 4.90 & 4.87 & 4.79 \\
\hline
\end{tabular}




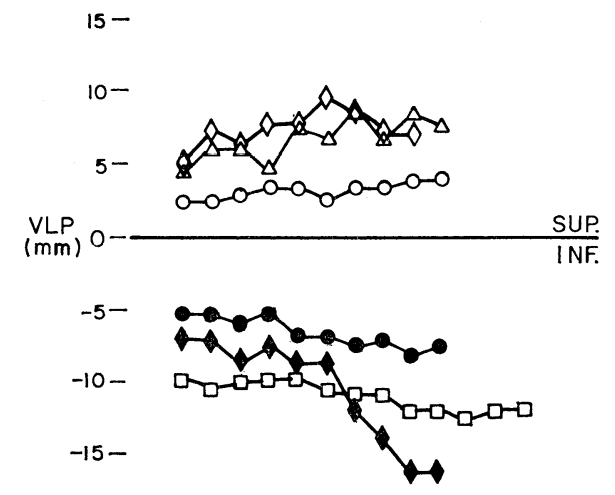

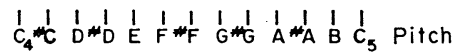
$262277294311320349370392415440466494323 f_{0}(H z)$

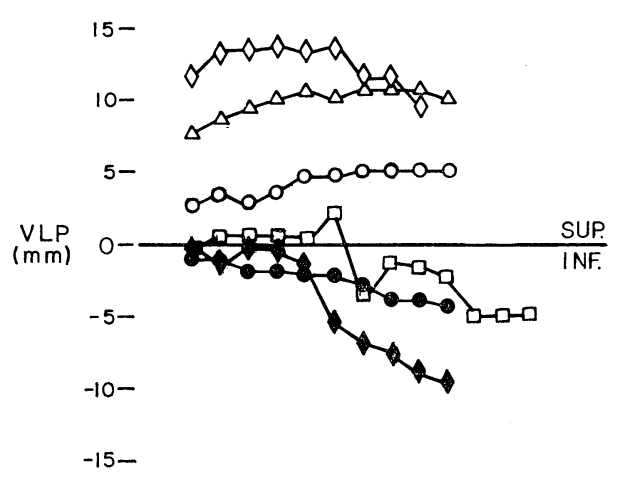

$$
\begin{aligned}
& \begin{array}{lllllllllllll}
1 & 1 & 1 & 1 & 1 & 1 & 1 & 1 & 1 & 1 & 1 & 1 & 1
\end{array}
\end{aligned}
$$

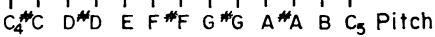

$$
\begin{aligned}
& 262277294311320349370392415440486494523 f_{O}(\mathrm{~Hz})
\end{aligned}
$$

Fig. 1 Measures of superior or inferior vertical larynx position (VLP) with respect to the physiologic rest position (zero line) of four singers producing sustained phonation at selected fundamental frequencies $\left(F_{0}\right) . \quad \triangle$ Subject A (early music singing voice), $\bigcirc$ Subject $B$ (Chinese singing voice), Subject B (Western opera singing voice), $\diamond$ Subject $C$ (early music singing voice), Subject $C$ (Western opera singing voice), $\square$ Subject D (Western opera singing voice).

were found in the spectra of not only the Western operatic voices, but also the Chinese and early music singing voices in this study. Those peaks were found roughly between 1.8 and $3.8 \mathrm{kHz}$, which we shall call the Bright timbre Frequency Range, or BFR (see Figs. 2 4). For each individual, the maximum

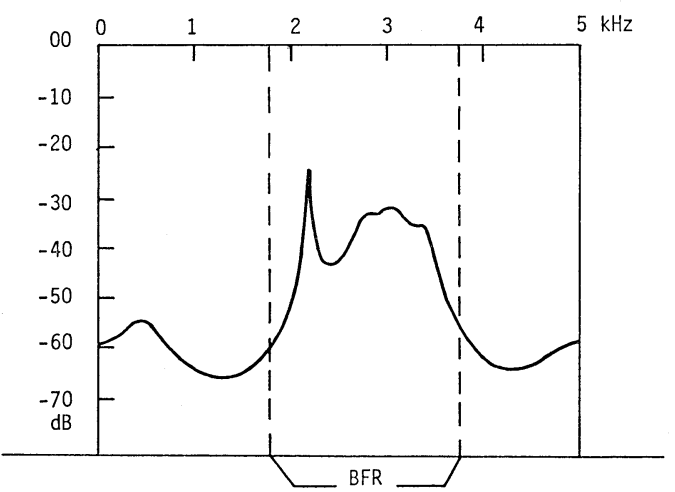

Fig. 2 A Chinese singing voice. vowel: /i/, pitch: \# $C_{4}(277 \mathrm{~Hz}), \mathrm{LPC}$ spectrum.

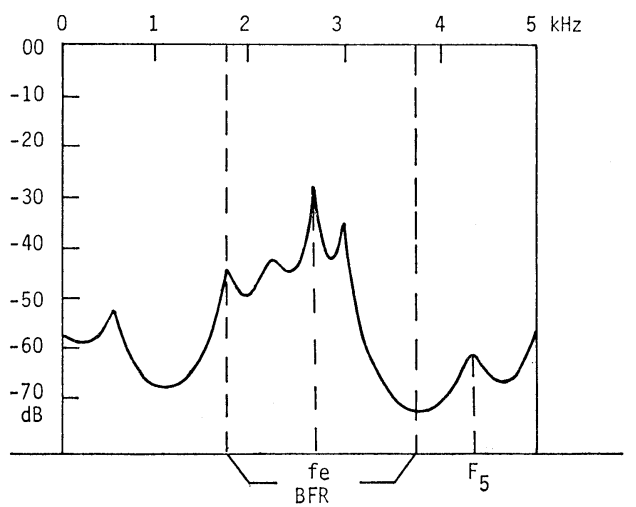

Fig. 3 A Western opera voice. sung vowel: /i/, pitch: $\# C_{4}(277 \mathrm{~Hz}), \mathrm{LPC}$ spectrum.

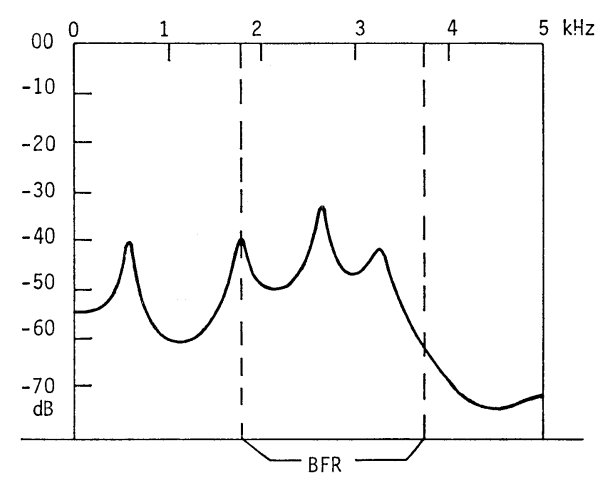

Fig. 4 An early music singing voice. vowel: /i/, pitch: $D_{4}(294 \mathrm{~Hz})$, LPC spectrum.

bandwidth between the centre frequencies of those peaks is roughly less than $1.5 \mathrm{kHz}$. The peaks were generally compact and higher in amplitude than the high formants in the speaking voice. Usually $F_{5}$ was 
not close to those peaks.

6) In both high and low laryngeal singing, the relative amplitude of the formants in the BFR increased as the fundamental frequency increased.

7) Generally there were more formant peaks with stonger relative amplitude in the $\mathrm{BFR}$ and weaker $F_{1}$ on /i/ spectrum than on /u/, with /A/ lying between them (see Figs. 3, 5, and 6), especially for the same subject and singing style.

8) All the formants frequencies were higher for the Chinese and early music voices when compared to the Western opera voice in the experimental range $\left(C_{4}-G_{5}\right)$ with three samples per half pitch step. These measurements were taken on two subjects, each singing in two styles, and generally hold true

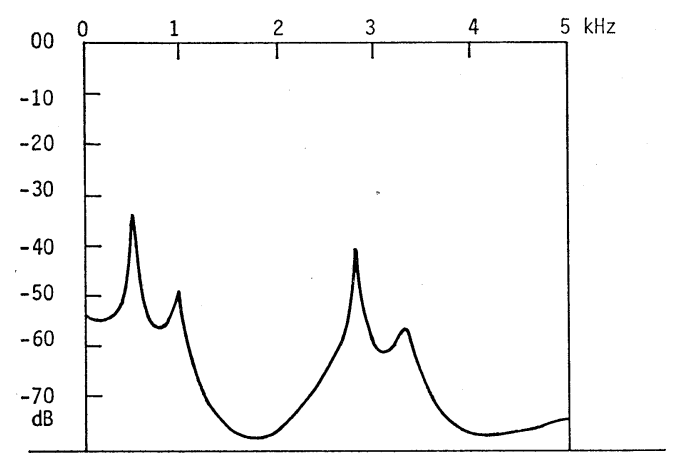

Fig. 5 An early music singing voice. sung vowel: /u/, pitch: $C_{4}(262 \mathrm{~Hz}), \mathrm{LPC}$ spectrum.

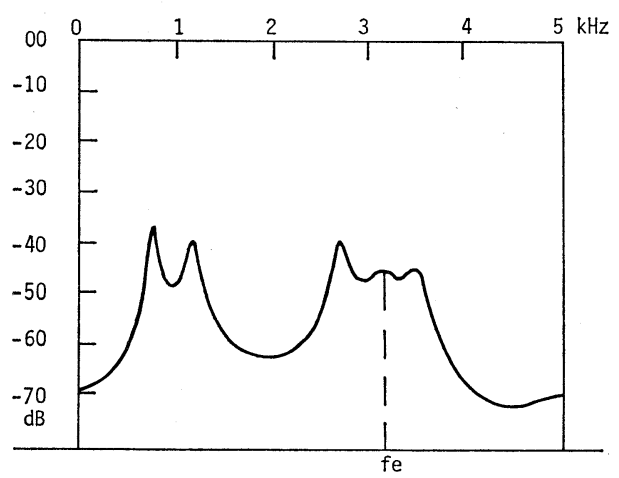

Fig. 6 A Western operatic voice. sung vowel: /A/, pitch: $F_{4}(349 \mathrm{~Hz})$, LPC spectrum.

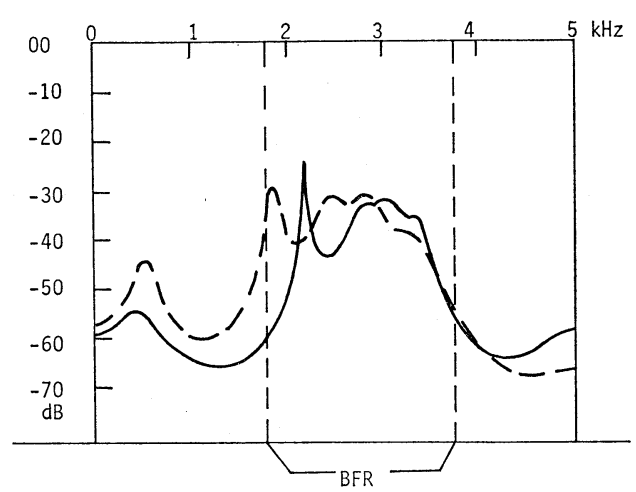

Fig. 7 - - - A Western operatic voice. A Chinese singing voice. vowel: /i/, pitch: $\# C_{4}(277 \mathrm{~Hz}), \mathrm{LPC}$ spectrum.

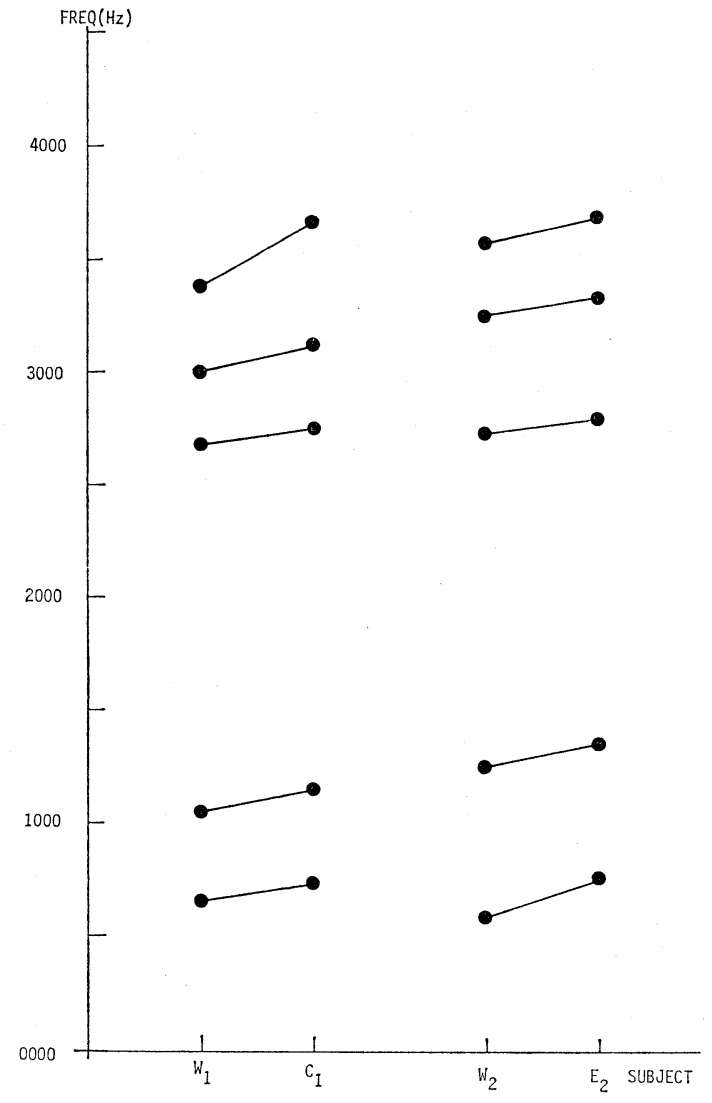

Fig. 8 Comparisons of singer's formants in different styles. vowel: /A/, $W_{1}$ : Western operatic voice of subject $1, C_{1}$ : Chinese singing voice of subject $1, W_{2}$ : Western operatic voice of subject $2, E_{2}$ : early music voice of subject 2 . 
except for $F_{1}$ in the vowel /i/in which a slight inverse change occurred (see Figs. 7 and 8).

9) For the same subject and same vowel phonation most formants of low laryngeal (the Western operatic) singing voices, except for some cases, were generally lower than those of the speaking voice. However in high laryngeal singing (the Chinese and early music singing) voice the formants roughly tended to shift upward, except $F_{4}$ which was usually compacted to $F_{3}$ and $f_{\mathrm{e}}$. The shifting direction of the formants in high laryngeal singing is not as consistent as that found in low laryngeal singing.

10) Frequently in the spectra of the Western operatic voice five or six formants were present, but in Chinese and early music singing voice there were four or five formants. In both cases the high formants, except $F_{5}$, generally were compacted (see Figs. 2 and 3). Also, the extra peak did not always appear.

\section{DISCUSSION}

Here we restate some points of this author's previous presentations, ${ }^{14-16)}$ to extend the discussion on the singer's formant.

1) Although both high and low laryngeal styles of singing are capable of producing bright voice, there are differences in timbre perceptible to trained ears as we know from voice experience. The difference of timbre between high laryngeal singing (open) and low laryngeal singing (covered) relates also to phonetic (vowel) modulation caused by articulation. For example, the $/ \mathrm{A} /$ sung by covered technique in high pitch is not a pure /A/ but a "hybrid" vowel of $/ \mathrm{A} /$ and $/ \mathrm{o} /$, (or $/ \mathrm{A} /$ and $/ \mathrm{u} /$ ). Therefore the formants, especially, the first and second formants $\left(F_{1}\right.$ and $\left.F_{2}\right)$ are correspondingly affected by phonetic (vowel) modulation. In this paper we mostly discuss the singer's high formants, and we will present and discuss the results of singer's low formants in another paper.

2) This study does not confirm previous findings that singing with a high larynx is a characteristic that distinguishes an untrained singer from a trained one. This result indicates that singing with a high larynx, in and of itself, does not necessarily produce a voice of poor quality, or cause poor vocal health. Although we know from voice training that there are many untrained, or poorly trained singers who raise their larynges in singing, the mechanism of singing with a high larynx used by some well-trained singers must be different from them. In this research I also analyze some spectra of untrained voices produced with a high larynx. The amplitude of their high formants are low or unstable. Further determination of the physiological and acoustic differences between good and bad singing with a high larynx would be an interesting topic for future research; the results could have important implications for the training and care of the professional voice.

Because the singing voices were rated as bright and we also found the extra formant $f_{\mathrm{e}}$ in high larynx singing, the explanation of Sundberg and Bartholomew, based upon studies of the Western operatic voice, that larynx lowering is responsible for the singing formant and bright timbre, does not acount for their presence in this study. Therefore, lowering the larynx is not necessary to produce bright timbre and its associated acoustic features. It seems that generally the lower the larynx and the wider the pharynx, the lower most formants are likely to be and the easier it is to associate a voice with dark timbre. Moreover, in voice training we know also that one way voice students begin to learn covering is often by lowering the larynx and expanding (directly or indirectly) the pharynx. Usually the voice is dark without much brightness. This step is not very difficult for them to achieve. It is only in the advanced stage of voice training, i.e., when the singer can open the pharynx properly and coordinate this with articulation of the whole vocal tract including the front cavity and also with the breathing, that the singer can achieve the covered bright voice. This step however is a very hard one for them to achieve. Perhaps, the physiological adjustment in covered singing technique that causes bright timbre and its associated acoustic features is something much more complex than the lowering of the larynx. Further and in-depth research is required to discover the actual physiological mechanics in the production of bright covered voice.

Now that trained singers with either high or low laryngeal singing technique can produce quality voice and have its corresponding featured formant, the lowering larynx therefore would not be able to reveal the essence of the production for the professional singing voice. In fact many singers just keep their comfortable and relatively stable laryngeal position (of neutral, low or high) to develop quality voice in the various styles. This is popular and effective in voice training as well. 


\section{S. WANG: SINGER'S HIGH FORMANTS}

However, from the results of our experiments, a lowered larynx position can be yet regarded as a distinctive feature for the covered singing voice, though not for the trained voice. Therefore, this physiological feature could be a clue that leads us to explore the singing technique associated with the production of covered timbre.

3) The study showed that in different styles of singing the high formants pattern varied but all had similar characteristics. The two to four very high and often compact energy envelope peaks including a possible extra peak roughly between 1.8 and $3.8 \mathrm{kHz}$ (BFR) would be the correspondingly similar acoustic features in the spectrum, and thus could be called the singer's (high) formant. We may believe that if there is an extra formant $f_{0}$ between or near $F_{3}$ and $F_{4}$ it could enhance the compactness and thus the amplitude of the peaks $F_{3}$ and $F_{4}$, and increase the sense of brightness. However the bright singing voice can also correspond to three or even two of those formants without the extra formant $f_{\mathrm{B}}$ (see Fig. 4). The frequency range $(1.8 \sim 3.8 \mathrm{kHz})$ of the variations on the common pattern of the singer's formant falls within the most sensitive frequency range $(1 \sim 4 \mathrm{kHz})$ of the human ear. This characteristic of singer's high formant combined with that of low formants for a singing voice might cause the voice to sound brighter and louder, and so please the human ear.

4) In spectra analysis, we found that in the low laryngeal singing (covered) voice more often there were 5 or 6 formants; however, in the high laryngeal (Chinese and early music) singing voice more often there were 4 or 5 formants. Here we need to explain the number of the formants and the compactness of the high formants in the spectra of the different singing voices. In fact we have to sublate the theory of lowering the larynx and then the extra formant $f_{\mathrm{e}}$ could be used for interpreting the different patterns of singer's formant in both high and low laryngeal singing.

In the covered voice lowering the larynx causes the lengthening of the vocal tract which in turn causes most formants of singing generally to shift downward from the corresponding formants of the speaking voice. Therefore under the cutoff frequency $(5 \mathrm{kHz})$, there could be five normal shifted formants plus the extra formant $f_{\mathrm{e}}$, thus six formants in total-that is, $F_{1}, F_{2}, F_{8}, f_{\mathrm{e}}, F_{4}, F_{5}$, in which $F_{3}, f_{\mathrm{e}}, F_{4}$, are compacted owing to the insertion of $f_{\mathrm{e}}$ between or near $F_{3}$ and $F_{4}$ (see Fig. 3). However in the high laryngeal singing voice the raising of the larynx causes the vocal tract to shorten which in turn causes some formants of the singing voice to shift relatively upward if the larynx position of the speaking voice is lower than that of the singing voice. In this case the high formant $F_{5}$ may shift over the cutoff frequency so that we only have the first four normal formants plus an extra formant $f_{\mathrm{a}}$, thus five formants in total-that is, $F_{1}$, $F_{2}, F_{3}, f_{\theta}, F_{4}$, in which $f_{8}$ is between or near $F_{3}$ and $F_{4}$ (see Fig. 2). Therefore $F_{3}, f_{\mathrm{e}}, F_{4}$, are compact and enhance each other in amplitude so that this five formants' pattern differs from that in the normal speech spectrum. Sometimes in high laryngeal singing, for example, when the larynx is only raised a small bit (yet higher than the larynx position in the corresponding speaking phonation), and owing to some compensation of articulation, $F_{5}$ is shifted up but is still under the cutoff frequency so we could also have six formants, that is, $F_{1}, F_{2}, F_{3}, f_{e}, F_{4}, F_{5}$ (see Fig. 9), which for the same above-mentioned reasons are compacted and enhanced in amplitude. Usually, $F_{3}$ is not very close to the compact $F_{\mathrm{a}}, f_{\mathrm{e}}$, $F_{4}$, has a low amplitude, and thus does not appear as consistently as $F_{8}, F_{4}$, as in the similar case with the speech spectrum. So, generally we found five formants instead of six formants, but these five formants are different from those in the speech spectrum. Here in the high laryngeal singing voice the five formants are $F_{1}, F_{2}, F_{8}, f_{\theta}, F_{4}$ (see Fig. 2), but in the speaking voice the five formants are $F_{1}, F_{2}, F_{3}, F_{4}$, $F_{5}$. Owing to the difference of the compactness and the amplitude of the high formant peaks between the two cases, the compact $F_{3}, f_{\mathrm{e}}, F_{4}$, in the singing spectrum is obviously distinguishable from $F_{3}, F_{4}, F_{5}$ in

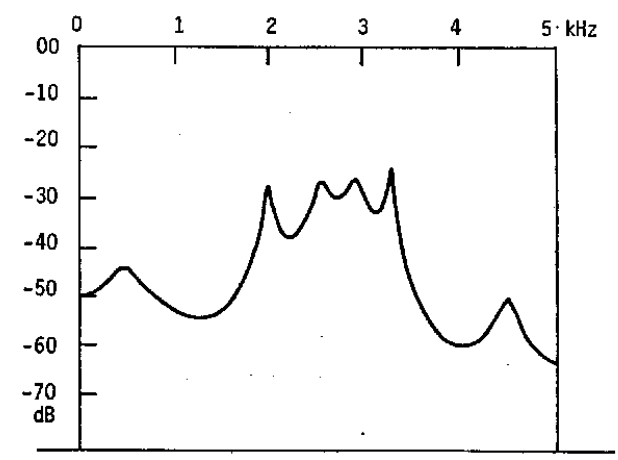

Fig. 9 Chinese singing voice. sung vowel: /i/, pitch: $E_{4}(330 \mathrm{~Hz}), \mathrm{LPC}$ spectrum. 


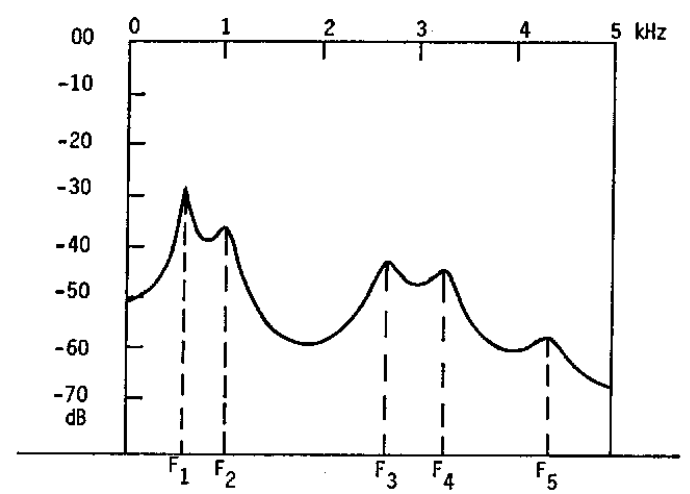

Fig. 10 Speaking phonation /A/, fundamental frequency $131 \mathrm{~Hz}$, LPC spectrum.

the speaking spectrum (see Figs. 6 and 10) though both of them have the same number of formants.

Moreover, sometimes there are only two formants in the BFR. It may mean that no extra formant is produced. That is, on the spectrum of singing voice the formant could be $F_{1}, F_{2}, F_{3}, F_{4}$ or $F_{1}, F_{2}, F_{3}, F_{4}$, $F_{5}$. In these cases, the high formants are not very compact but have a higher amplitude (see Figs. 4 and 11) that make the singer's high formants differ from speech high formants. However, for some singers, if the manner of speaking is similar to that of the singing voice, the spectra of the speaking and singing could also be similar. For example, among our subjects, one's speech was very much like his singing ("focused" and bright), and the singer's formant (even including the $f_{\mathrm{e}}$ ) was found in his speaking spectrum (see Figs. 6 and 12). In this sense, there is not an absolute boundary between them. So the formant patterns, or the acoustic features in radiated spectra between singing and speaking, are generally different, but similar and uniform under special conditions. Perhaps this is a kind of acoustic explanation for the saying "singing is the extension of speaking".

We found that in the BFR for /i/ spectrum there were more peaks than for the $/ \mathrm{u} /$ spectrum. It seems that on the spectra of the $/ i$ / singing phonation, the extra formant occured more often than that of $/ \mathrm{u} /$. We are also aware that generally the larynx position when singing /i/ was higher than that when singing $/ \mathrm{u} /$ ! (See Fig. 1). Moreover for $/ \mathrm{i} /$, the frequency of $F_{2}$ was very high and close (even it joins) to the compacted (and separate) $F_{8}, f_{\mathrm{e}}, F_{4}$ in BFR, and they enhanced each other, causing a high amplitude.

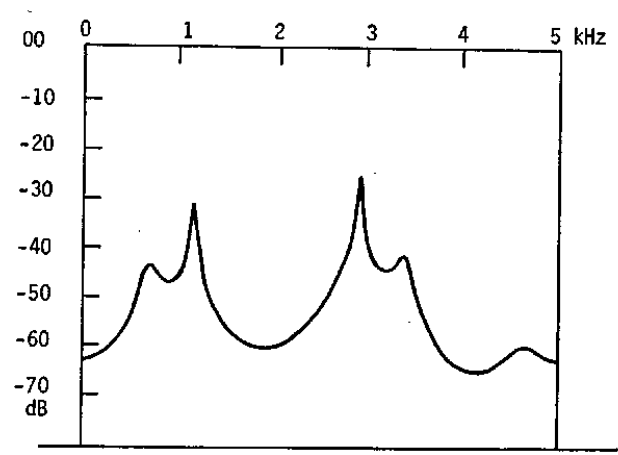

Fig. 11 Sung vowel $/ \mathrm{A} /$, a Chinese singing voice, pitch: $C_{4}(261 \mathrm{~Hz}), \mathrm{LPC}$ spectrum.

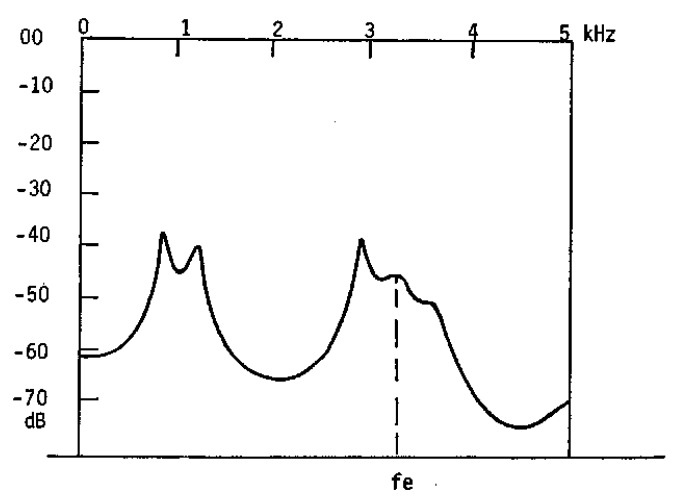

Fig. 12 A Western opera singer. speaking phonation $/ \mathrm{A} /$, fundamental frequency $128 \mathrm{~Hz}$, LPC spectrum.

Perhaps, this is a good reason why /i/ (and /A/) generally sound brighter than $/ u /$ in singing (and in speaking also). On the other side since $/ \mathrm{u} /$ has more stronger formants in the lower frequency range (under $1 \mathrm{kHz}$ ) than $/ \mathrm{i} /$ or $/ \mathrm{A} /$ then it sounds darker than them.

From the results and discussion above we know that the extra formant could also appear in high laryngeal singing. We may assume that the area mismatch and the separate larynx resonator (as stated in Sundberg's explanation) could happen in high laryngeal singing. This result and assumption made the maximum area ratio $1 / 6$ questionable. It seems necessary for future discussion to have a further investigation of the actual ratio value of the area in both high and low laryngeal singing, and of the maximum ratio within which condition the larynx 
tube separates from the remainder of the vocal tract.

So far we have explained several cases of singer's high formant in high and low laryngeal singing. In fact the singer's formant patterns can be more varied and complex than the above-mentioned cases, and affected by many factors. Because the extra formant determined by the independent larynx tube is not always located between $F_{3}$ and $F_{4}$, and $F_{5}$ does not always show up, the pattern of the singer's high formant in the spectrum sometimes is very intricate. The rounding or spreading and the protuding or withdrawing of the lips effect the length of the vocal tract quite a bit-this is another reason that sometimes we can not entirely explain the complex shifting of the formant only by the change of the larynx location. In low larynx singing if the length of the rest of the vocal tract except the independent larynx tube is shorter than that of the corresponding speaking vocal tract the shifting of the formants in the singing voice will not be as simple as what Sundberg described. All the above factors have to be considered when analyzing spectra. Moreover the modification of the vowel, the compensation and predominance of articulation, the morphology of the individual vocal tract, etc., greatly effect the formants, especially the two low formants $F_{1}$ and $F_{2}$.

5) It is noticed that Sundberg ${ }^{4,5,13,20,21)}$ changed the meaning of the singing formant. At first, Sundberg ${ }^{13)}$ wrote "The singing formant, located in the frequency range $2.5 \sim 3.0 \mathrm{kHz}$ is strongly dependent on the larynx tube. Actually, the larynx tube appears to behave as a Helmholtz resonator for the fourth formant." According to his explanation, ${ }^{4,5)}$ the singing formant, as the frequency of the independent larynx tube, should equal $F_{4}$. Recently Sundberg ${ }^{20,21)}$ has asserted that the "singing formant or the singer's formant, as I prefer to call it today, ... is constituted by a clustering of $F_{3}, F_{4}$, and $F_{5}, \ldots$ and this cluster can be explained as an extra formant associated with the larynx." [Sundberg ${ }^{13)}$ also said that the singing formant "seems to consist of two or three formants." This definition of his would not be compatible with his explanation of $F_{4}{ }^{13)}$ and $f_{\mathrm{e}}{ }^{4,5)}$ associated with a separate larynx tube. Now that he has changed it to "a clustering of $F_{3}, F_{4}, F_{5}$," we only discuss here his new one.] Here logically we understand Sundberg's definition of singing formant as follows: First, in Sundberg's terms singing formant and singer's formant are the same. Second, his concept of the singing formant and its frequency which is determined by the separated larynx tube resonator actually has three different definitions and values: (1) $F_{4}$; (2) the extra formant $f_{\mathrm{e}}$ which, as mentioned in the introduction, is between but separate from $F_{3}$ and $F_{4}$; (3) a cluster of $F_{3}, F_{4}$, $F_{5}$. The concept of the singing formant is confused not only by changing meanings but also by the principles of acoustics. In fact Sundberg's recent definition of singing formant, that is the cluster of $F_{3}, F_{4}, F_{5}$ appears not only inconsistent but also incompatible with his explanation. From the acoustic viewpoint of voice production $F_{3}, F_{4}$, and $F_{5}$ cannot all be producted by this separate larynx tube. Moreover, this definition would mean that $F_{3}, F_{4}$ and $F_{5}$ would be regarded as one formant, and therefore, there would be only three formants in total in the singing spectra (i.e., $F_{1}, F_{2}$ and the cluster). Obviously this is not an inclusive picture of the various patterns of the singer's formant as we have shown in the figures, especially, as $F_{5}$ usually is not close to the cluster $\left(F_{5}-F_{3}\right.$ can be as big as 2 to $2.5 \mathrm{kHz}$ ).

Today Sundberg's terms (singing formant and singer's formant), and the meanings ( $f_{\mathrm{e}}$ and the cluster of $F_{3}, F_{4}, F_{5}$ ) seem confused or appear vague as used in the literature. For example, the meaning used in singer's formant in SMAC 83 Proceedings and in The Science of Sound ${ }^{22)}$ seem different. It is necessary to clarify the concept of Sundberg's singer's formant for better understanding and future discussion. Since the extra peak $f_{\mathrm{e}}$ (other than $F_{3}$ and $F_{4}$ ) does not always appear in the spectra of singing voice it would not be proper to consider it a criterion of voice quality (evaluation) and to use it as the singer's formant to generalize the acoustic feature in the radiated spectrum of singing voice. On the other hand, because sometimes the extra peak $f_{\mathrm{e}}$ appears, and $F_{5}$ does not always appear (such as in the abovementioned high laryngeal singing, the $F_{5}$ could be shifted over the cutoff frequency, and even when $F_{5}$ appears it is not usually close to the cluster $F_{3}, f_{\mathrm{e}}$, $F_{4}$ ), it does not seem proper to use a cluster of $F_{3}$, $F_{4}, F_{5}$, as the definition of the singer's formant either. Otherwise, not only would they ignore the variety of formant patterns of the singing voice which characterize individual voice timbre but would put forth the questionable conclusion that the soprano voice has "no singing formant" (although to date we have not developed an accurate means of measuring the formants of the female voice in high fundamental frequency). During singing a soprano does adjust the 
shape and length of her vocal tract, and therefore the corresponding acoustic features (natural frequencies) of the modulated vocal tract must exist (some researchers ${ }^{18)}$ also reported some findings of singer's formant in soprano singing voice though their concept about singing formant is a bit different). This author also analyzed some tenors' falsetto voice in whose spectra there does not seem to be very high energy peaks in the BFR. It is known ${ }^{3,22,23)}$ that the male full voice is produced mainly with the heavy mechanism and the male falsetto voice with the light mechanism. The soprano (especially in high pitch) uses light mechanism (mixing some heavy mechanism in singing). Apparently it is the vibrating mode of the vocal cords (asociated with certain adjustments of supra/sub glottal systems) rather than the voice type or the sex of the singer that effect the amplitude of the high harmonics and thus of the high formants, and so effect the singer's formant. Moreover, since the amplitudes of all the formants affect the dynamic level of the singing voice the singer's (high and low) formants also do.

Sundberg's concept and explanation of singing formant due to the lowered larynx was limited to male voice, and he ${ }^{4,5)}$ has a separate and different conclusion and explanation for the female voice. Now in some soprano voices Sundberg has found raised larynges during singing. This could also raise a logical inference question: Would this new finding in sopranos be compatible or irrelative to Sundberg's lowered larynx explanation for the singing formant in the male voice, ${ }^{20)}$ to the conclusion that soprano has no singing formant, and to his former conclusion ${ }^{13)}$ that "singing with a high larynx may damage the vocal cords"?

Based upon the results of this study and combined with some results of other researchers (including Sundberg), the definition of the singer's formant proposed in point 3 of this paper would not have the above problems, but comments and criticism are expected and welcomed.

6) In line with the explanation of the lowered larynx, Sundberg ${ }^{4,5,24)}$ claimed that the formant in the singing voice shifted downward all formant frequencies other than the larynx-dependent extra formant relative to the corresponding formants in the speaking voice. Sundberg ${ }^{25)}$ concluded that "the first order effect on the formant frequencies of a shift in the larynx position stems from the shortening/ lengthening of the pharynx cavity. The effects on the formant frequencies of a raised larynx are, ... a combined rise in several vowels of the third and fourth formant frequencies." However, in this research for the professional high laryngeal singing voice, generally the formants except $F_{4}$, tend to be shifted upward relative to the corresponding formants in the speaking voice. The shifting direction of formants in high laryngeal singing are not as consistent as those in low laryngeal singing. One explanation for this could be that the vowel modification in high laryngeal singing is not as great as that in covered (i.e., low laryngeal) singing. Another explanation for this could be that the larynx position when speaking is not usually the physiological resting position, and is mostly higher than the physiological resting position. Therefore the laryngeal location difference between high laryngeal singing and speaking is not as large as the difference between lowered laryngeal singing and speaking. Sometimes the larynx location of speaking was found to be even higher than that of high laryngeal singing for some subjects, especially in the middle range. So the direction (upward/downward) of the shifted formants in singing vs. speaking roughly depends on the relative location of the larynx in singing vs. speaking. That is, the shifting direction of the formants could roughly coincide with the direction of the larynx from speaking to singing. We should also notice that $F_{4}$ in high laryngeal singing is not likely shifted up from that in speaking. Clearly the $F_{4}$ behavior in high laryngeal singing could not generally be explained by the larynx position, i.e., the shortening of the length of the vocal tract. So compensation of articulation and other reasons should be considered in order to explain the frequency difference of formants in singing and speaking. Another example: for the low formants $F_{1}$ of $/ \mathrm{i} /$ in covered (low larynx) singing is actually higher (rather than lower) than that in the corresponding speaking. Obviously this can not be explained by the lengthening of vocal tract. Perhaps, this is predominantly caused by vowel modification. That is, $/ \mathrm{i} /$ is modified by $/ \mathrm{u} /$. We know that the $F_{1}$ of $/ \mathrm{u} /$ is higher than $/ \mathrm{i} /$ in speaking voice. So the $F_{1}$ of /i/ in covered singing voice was shifted up. It seems that the shifting of low formants from speaking to singing is sensitive to the change both of the shape and length of the vocal tract, and that of high formants to the change of the length, i.e. mainly by the larynx position.

7) We know that the function and characteristics of voice source and the interactions between the 
source and the supra/sub glottal system are very important in affecting voice quality, especially in high voice. In voice training, in fact one of the main tasks for the singer is to discover the sense of proper interactions between the larynx and breathing (subglottal system), and between the larynx and articulation (supra glottal system) in order to be capable of producing a good voice by correctly handling the relationship.

The different laryngeal locations during phonation in different styles of singing perhaps means different functions or characteristics of voice source; in high laryngeal singing, the voice source adjustment used by well trained singers and untrained singers may also be different. For example, since the amplitude of high formants of good high laryngeal singing voices is high, by inference, the harmonics and so the fundamental, in their voice source must be also strong in amplitude. Therefore the physiological mechanism of the voice source for them is possibly different from that for poor high laryngeal singing. The relationship between the source and supra/sub glottal systems (such as lung pressure, glottal maximum opening, closure speed, degree of discontinuity at closure together with articulation, moreover, glottal friction and inductance, etc.) might vary with different styles, types, and even individual singers, and then, the approach for handling this relationship should also be different. That might be one of the reasons why we can find good and bad singing in either high and low laryngeal singing, and so laryngeal height should not be considered a reliable means of distinguishing good from poor singing voices. Further investigation of the voice source and the interactions between the laryngeal adjustment with supra/sub glottal systems will shed insight into the physiological mechanism and acoustic features of singing voice associated with different larynx position.

\section{CONCLUSION}

Professional singers produce singing voice in style associated with different laryngeal height. The singer's (high) formant patterns are varied but have similar characteristics. The extra formant is also found in the high laryngeal singing voice, and it is not a consistent acoustic feature in all professional singing voices. Lowering the larynx does not explain the extra formant, singer's formant and voice quality (brightness and evaluation). Sundberg's two definitions for singer's formant are not inclusive for the various formant patterns of singing voice associated with different laryngeal location and types. The low laryngeal position still can be regarded as a distinctive feature for the covered voice though not for the trained voice; the source characteristics, and the means of handing the relationship between the source and the supra/sub glottal systems are possibly different in styles of singing associated with high/low laryngeal singing.

\section{ACKNOWLEDGEMENTS}

The author expresses thanks to his former advisers and colleagues at the Speech Communication Group of Research Laboratory of Electronics at the Massachusetts Institute of Technology, and his colleagues at the Physics Department and the Music Department, York University. Special thanks are due K. Stevens, G. Fant, O. Brown, J. McKay and J. Burt. Gratitude is expressed to W. Wang, and K. Komar, to singers in China, Norway and the USA who were the subjects of this study, and to nineteen teachers and singers who served as jury in the perceived timbre study. Many thanks go to R. Kelly, C. Sharpe and R. Witmer.

The research and presentation were supported in part by the People's Republic of China and the (US) Voice Foundation, York University, and Brown Voice Seminar.

\section{REFERENCES}

1) W. Bartholomew, "A physical definition of good voice quality in the male voice," J. Acoust. Soc. Am. 6, 25-33 (1934).

2) S. N. Rzhevkin, "Certain results of the analysis of a singer's voice," Sov. Phys. Acoust. 2, 215-220 (1956).

3) W. Vennard, Singing: The Mechanism and the Technic (Carl-Fisher, New York, 1967).

4) J. Sundberg, "Articulatory interpretation of the singing formant," J. Acoust. Soc. Am. 55, 838-841 (1974).

5) J. Sundberg, "The acoustics of the singing voice," Sci. Am. 3, 82-91 (1977).

6) W. T. Bartholomew, "The role of imagery in voice teaching," NATS, USA, Research Committee, No. 1 (1951).

7) T. Shipp and K. Izdebski, "Vocal frequency and vertical larynx position by singers and nonsingers," J. Acoust. Soc. Am. 58, 1104-1106 (1975).

8) W. Frummhold and G. Hoppe, "Tomographische Studien zur Funktion des menschlichen Kehlkopfes. 1. Mittellung: Unterschiede in der Stimmlippenmechanik," Folia Phoniatr. 17, 83-91 (1965); 18, 81-90 (1966). 
9) K. Faaborg-Anderson and W. Vennard, "Electromyography of extrinsic laryngeal muscles during phonation of different vowels," Ann. Otolaryngol. Reinlaryngol. Laryngol. 73, 248 ff (1964).

10) R. Luchsinger and G. E. Arnold, Lehrbuch der Stimm- und Sprach-Herkunde (Springer-Verlag, Vienna, 1959).

11) W. Ruth, "The registers of the singing voice," NATS Bull. May 2-5 (1963).

12) A. Soninen, "Paratasis-gram of the vocal cords and the dimensions of the voice," Proc. 4th Int. Congr. of Phonetic Science, The Hugue: Mouton (1962).

13) J. Sundberg, "Formant structure and articulation of spoken and sung vowels," Folia Phoniatr. 22 (1), 28-48 (1970).

14) S. Wang, "Bright timbre, acoustic features and larynx position. (video film)," 12th Brown Voice Seminar, Amherst, USA (1983); 14th Symposium: Care of the Professional Voice, Denver, USA (1985).

15) S. Wang, "The relationship between bright timbre, acoustic features and larynx position: a comparsion of Western operatic with Chinese and some Western early music singing styles" (1983) (This paper was presented at the 105th Conference of ASA and included in NATS Bull. Jan/Feb, USA (1985)).

16) S. Wang, An Introduction to Voice Production (QuFu Teachers College Textbook, Qu Fu, 1976) (in Chinese).

17) G. Troup and H. Luke, "Some radiological observation of vocal source-vocal interactions," SMAC83, Proc. of the Stockholm Music Acoustics Conference Publications, Royal Swedish Academy of Music, No. 46: 1 (1983).
18) W. Seidner, H. K. Schutte, J. Wendler, and A. Rauhaut, "Dependence of the high singing formant on pitch and vowel in different voice types," SMAC 83, Proc. of the Stockholm Music Acoustics Conference Publications, Royal Swedish Academy of Music, No. 46: 1 (1985).

19) A. Johnsson, J. Sundberg, and H. Wilbrand, " $X-$ ray study of articulation and formants frequencies in two female singers," SMAC 83, Proc. of the Stockholm Music Acoustics Conference Publications, Royal Swedish Academy of Music, No. 46: 1 (1983).

20) J. Sundberg, Letter to S. Wang, Sundberg also noticed Wang that he realized recently "that singing with a high larynx occurs in professional singing and does not have to be harmful to the voice function" (1984).

21) J. Sundberg, "The voice as a sound generator," Research Aspects on Singing Publications issued by the Royal Swedish Academy of Music, No. 33 (1981).

22) T. Rossing, The Science of Sound (Addison-Wesley, Reading Mass., 1982).

23) J. C. Wilcox, The Living Voice (Carl-Fisher, New York, 1945).

24) B. Lindbolm and J. Sundberg, "Acoustical consequences of lip, jaw and larynx movement," J. Acoust. Soc. Am. 50, 1166-1178 (1971).

25) J. Sundberg and P. E. Norstrom, "Raised and lowered larynx-The effect of vowel formant frequencies," Speech Transmission Lab. Q. Prog. Status Rep. 2-3, 35-39 (1976). 